Mémoires du livre

Studies in Book Culture

\title{
Lectures biographiques et consécrations croisées : pourquoi Chris Marker fait-il de Jean Giraudoux un « écrivain de toujours »?
}

\section{Hervé Serry}

Volume 7, numéro 1, automne 2015

Une fabrique collective du patrimoine littéraire (XIX ${ }^{\mathrm{e}}-\mathrm{XXI}^{\mathrm{e}}$ siècles). Les collections de monographies illustrées A Collective Factory of Literary Heritage (XIX ${ }^{\text {th }}-\mathrm{XXI}^{\text {st }}$ centuries): The Collections of Illustrated Monographs

\section{URI : https://id.erudit.org/iderudit/1035761ar}

DOI : https://doi.org/10.7202/1035761ar

Aller au sommaire du numéro

\section{Éditeur(s)}

Groupe de recherches et d'études sur le livre au Québec

\section{ISSN}

1920-602X (numérique)

Découvrir la revue

Citer cet article

Serry, H. (2015). Lectures biographiques et consécrations croisées : pourquoi Chris Marker fait-il de Jean Giraudoux un « écrivain de toujours "? Mémoires du livre / Studies in Book Culture, 7(1). https://doi.org/10.7202/1035761ar

\section{Résumé de l'article}

Cet article examine un moment important, mais peu analysé, de l'oeuvre de Chris Marker : la publication de son essai Giraudoux par lui-même (1952). Cet essai, dans le contexte singulier des années d'après-guerre en France, marquée par l'hégémonie intellectuelle de Jean-Paul Sartre et de ses proches, permet d'interroger les prises de position de Chris Marker. Pourquoi cette entreprise de patrimonialisation de Giraudoux est-elle menée à cette période? Que révèlent cette relecture de Giraudoux, alors un écrivain oublié, et sa réception par la critique, au regard du parcours de Chris Marker, et des positions de ses alliés d'alors, la revue Esprit et les Éditions du Seuil (l'éditeur du livre dans la série " Écrivains de toujours »)?
Tous droits réservés (C) Groupe de recherches et d'études sur le livre au Québec, 2016 cest protége par la loi sur le droit d'auteur. L'utilisation des services d'Érudit (y compris la reproduction) est assujettie à sa politique d'utilisation que vous pouvez consulter en ligne.

https://apropos.erudit.org/fr/usagers/politique-dutilisation/ 


\title{
MÉMOIRES DU LIVRE \\ STUDIES IN BOOK CULTURE
}

\section{LECTURES BIOGRAPHIQUES ET CONSECRATIONS CROISEES : pourquoi Chris Marker fait-il de Jean Giraudoux un « écrivain de toujours »?}

\author{
Hervé SERRY \\ UMR CRESPPA
}

Cet article examine un moment important, mais peu analysé, de l'œuvre de Chris Marker : la publication de son essai Giraudoux par lui-même (1952). Cet essai, dans le contexte singulier des années d'après-guerre en France, marquée par l'hégémonie intellectuelle de Jean-Paul Sartre et de ses proches, permet d'interroger les prises de position de Chris Marker. Pourquoi cette entreprise de patrimonialisation de Giraudoux est-elle menée à cette période? Que révèlent cette relecture de Giraudoux, alors un écrivain oublié, et sa réception par la critique, au regard du parcours de Chris Marker, et des positions de ses alliés d'alors, la revue Esprit et les Éditions du Seuil (l'éditeur du livre dans la série « Écrivains de toujours »)?

This paper explores a neglected but important dimension of the intellectual production of Chris Marker: the publication of his study of the French writer Jean Giraudoux (1952). Within the unique context of post-war France, marked as it was by the intellectual hegemony of Jean-Paul Sartre and of those around him, the book invites an examination of Marker's intellectual choices. Why did he undertake this patrimonial effort at that moment in time? What does his rereading of Giraudoux, an author who was practically forgotten by then, and of the critical reception of Giraudoux's work, bring to Marker's career and to the stance of his allies at the time, Esprit and Seuil, publishers of the Écrivains de toujours series?.

Vol. 7, $\mathrm{n}^{\circ} 1 \mid$ Automne 2015 
En 1952, Christian Marker ${ }^{1}$ signe dans la collection «Écrivains de toujours » des Éditions du Seuil un volume consacré à Jean Giraudoux ${ }^{2}$. Cette série, dont chaque volume est composé d'un essai introductif, d'une anthologie et d'une bibliographie ${ }^{3}$, repose sur le principe « d'une vulgarisation de qualité » qui doit "satisfaire ensemble une curiosité sur l'homme, le goût de la biographie et de l'anecdote, la curiosité sur l'œuvre: ceci pour le grand public populaire ${ }^{4} »$. Nous reviendrons sur l'intérêt de Marker pour la diffusion de la culture, qui croise celui pour l'art théâtral, apprécié dès l'adolescence, et dont Giraudoux apparaît comme un maitre. La collection vient d'être inaugurée : en 1951 ont été lancés les quatre premiers titres, quatre autres paraissent l'année suivante. Pour ses concepteurs, il s'agit de publier des écrivains « classiques » (Stendhal, Montaigne, Hugo...), mais aussi des contemporains (Colette et Giraudoux), afin de limiter la perception « scolaire » de la série. Par ailleurs, Chris Marker est un jeune auteur plein de promesses pour la maison d'édition naissante, et le publier de nouveau, après un roman d'aventures en 1949 (Le Cour net), est un enjeu qui démontre la volonté de l'attacher au catalogue.

Ce désir de faire figurer des auteurs d'une littérature contemporaine dans «Écrivains de toujours » recoupe celui de Christian Marker de redonner à Giraudoux un rôle majeur dans l'actualité littéraire des années 1950, importance qu'il avait eue dans les années 1930. À cette période, Giraudoux a connu la gloire littéraire, mais il est tombé, quelques années après son décès en 1944, dans une certaine invisibilité. Pour un critique théâtral de l'époque, Guy Dumur - par ailleurs un proche du Seuil -, «Giraudoux est de tous les écrivains français morts ou vivants le plus célèbre des inconnus ${ }^{5}$ ». Les raisons de ce déclin sont multiples. Comme nous l'évoquerons, son recueil, Pleins Pouvoirs (1939), puis les implications de l'écrivain dans le jeu politique - lorsqu'il devient Commissaire général à l'information et à la propagande en juillet 1939 -, ont contribué à cet oubli relatif. Celui-ci se renforce de l'hégémonie de la posture engagée de JeanPaul Sartre durant les années qui suivent le conflit, ce qui singularise encore la défense de Giraudoux par Marker.

Ce choix fait par les animateurs du Seuil et Christian Marker croise donc la stratégie éditoriale du Seuil, des enjeux biographiques et l'état des débats littéraires et politiques du début des années 1950. Marker ne se prive

Vol. 7, $\mathrm{n}^{\circ} 1 \mid$ Automne 2015

«Une fabrique collective du patrimoine littéraire (XIX ${ }^{\mathrm{e}}-\mathrm{XXI}^{\mathrm{e}}$ siècles). Les collections de monographies illustrées » 
d'ailleurs pas de mettre en scène ce croisement biographique entre lui-même et cet aîné admiré depuis de nombreuses années. Le titre de son essai introductif - «Giraudoux 52 », s'il joue aussi sur le rappel du titre de l'adaptation théâtrale du célèbre mythe, Amphitryon 38 - ainsi présenté par l'auteur comme la 38 e reprise -, est sans équivoque quant à l'inscription dans l'actualité que Marker opère puisqu'il s'agit de l'année de parution de l'ouvrage. Ce titre sera revu selon la date de chacune des rééditions du livre. Pour la seule traduction existante, en langue allemande, le titre devient « Giraudoux Heute » [aujourd'hui] ${ }^{6}$. Significative également, la première note de bas de page (qui disparait de l'édition allemande) explicite ce que Marker entend par «notre » Giraudoux. «Quand je dis "nous", je parle des garçons de ma génération (vingt ans en 1940) et particulièrement ceux qui ont bien tourné, qui pensent comme $\mathrm{moi}^{7}$. $\gg \mathrm{Si}$ la seconde partie de la phrase, et sa prétention, font réagir certains critiques - qui dénoncent une mode des jeunes critiques qui révèlent «à l'univers l'objet de leur admiration littéraire ${ }^{8}$ ", voire un certain $~ « \operatorname{dogmatisme} \mathrm{e}^{9} »-$, la première exprime l'inscription autobiographique de cet essai. De plus, Marker prend le risque d'évoquer Giraudoux à une époque et dans un contexte où l'on attend tout à fait autre chose de la littérature.

Pour tenter de saisir ces croisements, il est nécessaire, dans un premier temps, de situer Chris Marker en 1952 et de retracer brièvement sa jeune, mais déjà riche carrière intellectuelle. Considérer ce texte sur Giraudoux - et la manière dont il inscrit ce dernier dans le patrimoine littéraire - nécessite de partir de la trajectoire de Marker. Dans un deuxième temps, nous verrons la nature de sa réappropriation de Giraudoux, c'est-à-dire les manières dont il retraduit son inspiration pour l'adapter aux années 1950. Les réceptions critiques suscitées par cette relecture permettront de voir que l'implication forte de Marker afin de faire ré-exister Giraudoux, sous couvert d'un lien singulier entre l'œuvre et l'action, ne provoque qu'une attention restreinte et est diversement perçue à un moment où la politisation de l'espace intellectuel français atteint un sommet.

\section{Christian 21, 40, 45, 52...}

Innombrables sont les évocations de Chris Marker qui, quelle que soit leur nature, s'ouvrent par la mention des moyens divers mis en œuvre par cette

Vol. 7, n $1 \mid$ Automne 2015

«Une fabrique collective du patrimoine littéraire (XIX ${ }^{\mathrm{e}}-\mathrm{XXI}^{\mathrm{e}}$ siècles). Les collections de monographies illustrées » 
figure centrale des cultures visuelles, à la carrière multiple, pour brouiller les pistes autour de son itinéraire biographique ${ }^{10}$ - particulièrement pour les moments antérieurs à 1962, année de ses films Joli mai et La Jetée. En septembre 1949, alors qu'à la demande du Seuil il prépare le service de presse de son roman, Le Cour net, il souligne combien la notice biographique par lui communiquée à l'éditeur est en fait «superflue ». Il consent, si cela s'avère "vraiment indispensable ", à faire circuler une date et un lieu de naissance imaginaires - 22 juillet 1921 à l'Île aux Moines -, et comme seul "curriculum vitae "s'occupe de Culture populaire". Pour le reste, vous ignorez tout de moi, surtout mon adresse ${ }^{11}$.» Parmi les dispositifs qui fragmentent et réécrivent une biographie, le recours aux pseudonymes est un élément d'une certaine affirmation de l'autorité littéraire ${ }^{12}$. La liste des nombreux pseudonymes utilisés par Marker, dès ses années de jeunesse, se complète de présentations biographiques romancées, de son refus précoce des entrevues, autant d'éléments qui témoignent d'une attention singulière au récit des origines (d'une singularité légitime, bien entendu). Marker, à la fin des années 1990, revendiquait la mise à distance de ces «enthousiasmes de jeunesse ${ }^{13}$ ». Parmi ceux-ci, qui furent nombreux et éclectiques, une passion certaine l'emporte vers Jean Giraudoux, qui est un lien entre les deux moments de sa carrière. Cette admiration pour un écrivain mondain a pu étonner ses proches d'alors, comme le rapporte François Lecointe dans un texte d'hommage ${ }^{14}$. Dès son adolescence, Christian Bouche-Villeneuve fils unique d'une famille bourgeoise résidant dans une rue cossue de Neuilly-sur-Seine (le père de Marker travaille dans le secteur bancaire) cultive un vif intérêt pour la littérature ${ }^{15}$. Le peu d'intérêt qu'il manifeste pour les études, ses curiosités et ses aptitudes artistiques, ses «tenues étonnantes » frappent ses amis ${ }^{16}$. Avec des condisciples du lycée Pasteur de Neuilly, dont Bernard Voyenne et Bernard Pingaud, rejoints par des élèves du Cours secondaire tout proche, à l'instar de Simone Kaminker-Signoret, il participe à l'animation d'un modeste journal, Le Trait d'union. Sous pseudonyme, déjà, le jeune homme rédige des articles sur le théâtre, tout particulièrement sur les pièces de Giraudoux et de Marcel Achard. Certaines des œuvres théâtrales de ce dernier, auteur de comédies sentimentales, profitent durant les années 1930, comme celles de Giraudoux, des talents de Louis Jouvet, à une époque où l'art théâtral captive un public large et où chaque nouvelle pièce de Giraudoux est un évènement attendu.

Vol. 7, $\mathrm{n}^{\circ} 1 \mid$ Automne 2015

«Une fabrique collective du patrimoine littéraire (XIX ${ }^{\mathrm{e}}-\mathrm{XXI}^{\mathrm{e}}$ siècles). Les collections de monographies illustrées » 
Durant les "années noires", installé à Vichy où son père travaille pour le régime du Maréchal Pétain au sein de l'administration d'un ministère, il fonde, avec Bernard Pingaud et Pierre Kuentz, et dirige sous le nom de Marc Dornier, La Revue française. Cabiers de la Table ronde. Parmi les buts affichés, on peut lire dans la première des deux livraisons parues, qu'il s'agit de "créer un climat intellectuel favorable au redressement français", de "lutter pour la spiritualité", de permettre à la jeunesse de s'exprimer et d'agir ou encore de combattre «la stupidité, la bêtise, la facilité, le terre à terre, le snobisme... ${ }^{17} »$. Le contenu ne relève pas exclusivement des lettres et des arts, puisque sont publiés un message du commissaire général Jean Borotra promouvant l'éducation physique, une lettre de «Navarre» (le commandant Georges Loustaunau-Lacaud, responsable de «L'Ordre national») sur les causes de la défaite de juin 1940, ou encore un appel à «la révolution bancaire » par Georges Bouche-Villeneuve, le père de Christian. Néanmoins, cette participation au redressement de la France est d'abord artistique et spirituelle: Marc Dornier défend une «bonne» littérature, déliée de la propagande - il dénonce les livres d'Henry Bordeaux, proche de Pétain -, et la possibilité de « défendre la Pensée [car] c'est défendre la Vie », ou, autrement dit, "défendre la Pensée Française [car] c'est défendre la France ». Un troisième numéro sur la poésie, annoncé pour janvier 1942, ne paraittra pas et la revue, sans que l'on sache pourquoi, disparait. Si dans ses souvenirs Bernard Pingaud, qui évoque le choix vichyste de ses 18 ans, précise que «le fascisme était pour [lui], comme pour la plupart des jeunes gens qu[iil] fréquentai[t], l'emballage prestigieux sous lequel [ils] tent[aient] de faire passer une médiocre marchandise littéraire ${ }^{18}$ », en réalité la ligne de La Revue française est plus large.

Pour Marker, après cet engagement relevant d'une proximité (critique) avec le régime de Vichy, s'ouvre une autre époque. Quelques allusions et courts récits le décrivent au sein des Francs Tireurs Partisans, puis aux côtés de l'armée américaine durant des opérations liées au débarquement. C'est en fait un petit groupe d'amis qui se retrouvent dans ses engagements militaires ${ }^{19}$. Christian Bouche-Villeneuve séjourne en Allemagne, toujours sous l'uniforme de l'armée des États-Unis, d'où Pingaud le voit revenir en 1945 ou $1946^{20}$. Une courte notice biographique des éditions du Seuil précise le lien avec d'autres engagements alors que le conflit se termine :

Vol. 7, $\mathrm{n}^{\circ} 1 \mid$ Automne 2015

«Une fabrique collective du patrimoine littéraire (XIX ${ }^{\mathrm{e}}-\mathrm{XXI}^{\mathrm{e}}$ siècles). Les collections de monographies illustrées » 
«Ayant fait, pendant la guerre, ce qu'il avait à faire, s'est occupé à la Libération de culture populaire en France, puis en Allemagne ${ }^{21}$. »

En effet, avec Joseph Rovan, partisan dès la Libération d'un rapprochement franco-allemand - également défendu par les collaborateurs du Seuil et par ceux d'Esprit, dont Rovan est secrétaire de rédaction en 1945 et $1946^{22}$-, il anime Doc, un périodique des mouvements Peuple et Culture et Travail et culture, deux groupements d'éducation populaire dirigés par des militants de diverses obédiences religieuses et politiques. En 1947, des tensions éloignent Rovan et Marker de cette revue que des proches du Parti communiste entendent annexer, mais l'association Peuple et Culture, avec Rovan, Bégnino Cacérès et Joffre Dumazedier, poursuit son action et ses publications sont prises en charge par le Seuil.

Sans entrer dans le détail des engagements et des réseaux qui s'associent, Christian Bouche-Villeneuve, depuis La Revue française, évolue dans un milieu qui, à l'issue du conflit, se rassemble pour une part autour de la revue Esprit, d'Emmanuel Mounier, et des éditions du Seuil. En effet, le Seuil est fondé sur un programme intégralement catholique en février 1935. Sa production alors modeste est typique d'une effervescence intellectuelle catholique, en réaction, notamment, à cette «crise de civilisation» explicitée par Emmanuel Mounier et Esprit²3. Des temps troubles des années 1930 aux « années noires », les engagements se rejouent au sein d'un milieu militant relativement homogène. Ainsi de l'association Jeune France, dont Mounier fut en partie le concepteur, et Pierre Schaeffer ainsi que Paul Flamand, les animateurs. De 1940 à 1942, Jeune France est un bras armé de ce que l'on pourrait nommer la politique culturelle de la Révolution nationale ${ }^{24}$. Active sur de multiples terrains, Jeune France patronne notamment une séance de lecture théâtrale de La Revue Française, et des auteurs de la revue de Dornier/Bouche-Villeneuve sont des collaborateurs importants de l'association, à l'instar d'Alfred Cortot ${ }^{25}$.

Le Seuil est relancé par Bardet et Flamand en février 1943. Partageant la même volonté de préparer l'après-guerre, les animateurs de la petite maison d'édition et d'Esprit se rapprochent pour échafauder les conditions d'un travail commun. La paix advenue, de cette réflexion naît un accord d'édition qui voit le périodique personnaliste s'installer au Seuil. Un dispositif de trois

Vol. 7, $\mathrm{n}^{\circ} 1 \mid$ Automne 2015

«Une fabrique collective du patrimoine littéraire (XIX ${ }^{\mathrm{e}}-\mathrm{XXI}^{\mathrm{e}}$ siècles). Les collections de monographies illustrées » 
collections alimentées par des collaborateurs d'Esprit renforce cette alliance et procure à Bardet et Flamand un allié de poids. La fondation de la série «Écrivains de toujours » participe de cette dynamique de croissance, mais aussi de l'ambition du Seuil de ne pas être uniquement identifié à Esprit. Les affinités et les passerelles seront nombreuses entre les deux entités.

Chris Marker devient l'un de ces liens forts. En mai 1946, lorsque parait son premier texte dans la revue d'Emmanuel Mounier - «Les vivants et les morts ${ }^{26} »-$, ce dernier dépose sur le bureau de Paul Flamand des poésies du jeune auteur rassemblées sous le titre D'une planète morte et signées Chris Mayor. Après lecture, Paul Flamand refuse ce manuscrit traversé «d'une violence intérieure pleine de prix », mais encore trop vert ${ }^{27}$. C'est d'abord dans les sommaires d'Esprit que la plume de Marker est remarquée. On relève de très nombreuses contributions au «Journal à plusieurs voix » de la revue, qui autorise des formes et des sujets très divers, de l'actualité (parfois imaginaire) à la rêverie (parfois engagée). Quelle que soit la forme des textes ou le ton choisi (grave ou ironique), Marker est attentif aux questions sociales et politiques: il traite ainsi de la Résistance et du journalisme (livraison de mai 1947), de la guerre froide (mai 1947 et août 1948), du gaullisme de manière critique (novembre 1947), de la force émancipatrice du jazz (juillet 1948), du refus de l'anti-américanisme à l'occasion d'un numéro sur les nouvelles formes de diffusion culturelle (idem), ou encore, avec Henri Michard, il publie un très long "Documentaire » traitant de la «jeunesse délinquante » (juillet 1950). Marker mobilise ses proches, soit pour rendre compte de leurs œuvres, soit pour instaurer avec eux un dialogue, imaginaire ou pas, afin d'enrichir ses échanges avec les lecteurs. On retrouve parmi eux Bernard Pingaud, Alain Resnais, Bernard Voyenne, Franz Villier, ou encore le directeur d'Esprit rebaptisé Emmanuel Moonlight et dépeint comme un idéaliste ("Actualités imaginaires », avril 1947)... Les recensions d'ouvrages, les nouvelles, les prises de position esthétique (sur le cinéma, les dessins animés ou le genre policier) et les chroniques de Mayor, qui devient Marker, sont régulières pour la période de la fin des années $1940^{28}$.

Cette activité conséquente pour Esprit le rapproche du Seuil. Il s'intéresse à la production de la maison et, par exemple, partage l'enthousiasme de Pierre Schaeffer qui, dans Amérique nous t'ignorons (1946), livre un portrait vivant de ce pays $^{29}$. Il recommande des livres à Flamand et Bardet, effectue des

Vol. 7, $\mathrm{n}^{\circ} 1 \mid$ Automne 2015

«Une fabrique collective du patrimoine littéraire (XIX ${ }^{\mathrm{e}}-\mathrm{XXI}^{\mathrm{e}}$ siècles). Les collections de monographies illustrées » 
traductions pour eux ou encore leur donne un recueil de textes pour la jeunesse (L'Homme et sa liberté, 1949) et un roman d'aventures déjà évoqué (Le Cour net, 1949). Dans la continuité de sa participation active à Peuple et culture, il contribue à plusieurs livraisons de la collection éponyme publiée au Seuil (dont Regards neufs sur le mouvement ouvrier, 1952, et Regards neufs sur le cinéma, 1953). Un moment moins connu attire l'attention : sa participation à l'enquête commandée par le Seuil à l'abbé Émile Poulat au moment de la crise des prêtres-ouvriers. Alors que les tensions s'accumulent entre la hiérarchie de l'Église et ces prêtres militants qui travaillent dans les usines, il semble que Marker et Flamand participent durant plusieurs mois à la réalisation d'une compilation de documents autour de leur action, notamment sous la forme d'entretiens enregistrés. La direction du Seuil, dans une position incertaine lorsque l'Église, en 1954, lance aux prêtresouvriers un ultimatum pour qu'ils rentrent dans le rang de l'apostolat traditionnel, ne publiera pas le manuscrit pourtant achevé que Poulat propose à Jérôme Lindon des Éditions du Minuit.

On le voit, l'évocation des moments saillants des activités de Chris Marker relève d'implications en prise avec le monde social et intellectuel de son temps, au sein de multiples arènes. Cette présence est continue durant les années d'après-guerre, où l'horizon d'une possible reconstruction pacifique est à la fois traversé d'espoirs et d'inquiétudes, notamment en Europe. L'ensemble de ces prises de position de Marker suggère de percevoir l'essai sur Jean Giraudoux comme une date clé de ces engagements, à un moment de recherche d'une posture intellectuelle dont la résolution est fortement travaillée par la question politique.

\section{Les lectures possibles de Giraudoux dans les années 1950}

Alors que ce moment "d'invention de Chris Marker », selon l'expression de Catherine Lupton ${ }^{30}$, est encore loin de s'achever (avec l'entrée résolue dans les mondes de l'image), retenons d'abord que, pour certains commentateurs, l'idée de composer un portrait biographique de Jean Giraudoux en 1952 est une gageure. Dumur, cité précédemment, estime que si pour beaucoup d'auteurs la ligne des «Écrivains de toujours », visant à ce que ceux-ci soient "peints par eux-mêmes", semble aisée à suivre, elle pourrait s'avérer

Vol. 7, $\mathrm{n}^{\circ} 1 \mid$ Automne 2015

«Une fabrique collective du patrimoine littéraire (XIX ${ }^{\mathrm{e}}-\mathrm{XXI}^{\mathrm{e}}$ siècles). Les collections de monographies illustrées » 
difficile pour Giraudoux, peu disert sur lui-même, peu présent dans la vie littéraire et «cherchant toujours à se faire passer pour diplomate parmi les gens de lettres, et pour écrivain parmi les diplomates ${ }^{31} »$. Marker ne masque d'ailleurs pas ces difficultés qu'il évoque en débutant la partie anthologique de son livre : la discrétion de l'écrivain, voire «la plus délicieuse et irritante ambiguïté » des quelques pages de souvenirs qu'il a laissées, rendent difficile la rencontre du critique avec ce « spectre $^{32} »$.

Si, pour les concepteurs de la série, son «didactisme un peu cinématographique » permet «que se révèle le personnage de l'auteur ${ }^{33}$ », les manières de répondre à cet impératif sont variées d'un essai à l'autre. On peut relever, et les critiques ne manquent pas de le faire, que Marker s'implique personnellement dans son portrait: approche «sentimentale» relevée d'un mimétisme touchant pour l'un d'eux, essai brillant d'un jeune "diablement intelligent et habile», pour un autre ${ }^{34}$. Une recension souligne encore que la mort de l'écrivain semble être pour Marker une «cassure douloureuse, le grand évènement de la mort paternelle 35 ».

Ainsi, avec ardeur, dès les premières pages de son essai introductif, Marker évoque les souvenirs du groupe de lycéens auquel il appartient en 1937, spectateurs du théâtre du Cartel et convaincus que Giraudoux, Jouvet, Dullin, Pitoëff étaient leurs interlocuteurs directs, voire exclusifs, par-dessus la tête des bourgeois de l'orchestre qu'ils voient depuis les places bon marché. «Mais cette histoire de famille est une chose, et la survie littéraire en est une autre » : Marker veut montrer que Giraudoux résiste au temps et qu'une «nouvelle vie» s'ouvre pour lui aujourd'hui ${ }^{36}$. Selon la lecture d'André Bazin - collaborateur d'Esprit, mais aussi de Travail et Culture -, la méthode de Marker serait giralducienne, c'est-à-dire « étonnamment libre »: « il a l'air de nous entretenir de cinéma, de tragédie historique, de sa propre adolescence, il a l'air de dériver selon la séduction des thèmes apparus ou l'analogie des mots qui le font rebondir ${ }^{37} »$. Ici, la crainte, partagée par d'autres critiques, est de voir cette virtuosité inspirée se perdre au fil de l'œuvre de l'écrivain « simple amateur de $\operatorname{mots}^{38}$ », selon un reproche qui fut fréquemment adressé à Giraudoux.

Le travail d'explicitation mené par Marker débute par un constat : le célèbre écrivain et diplomate, qui fut premier en tout et de tous les classements ${ }^{39}$, est

Vol. 7, $\mathrm{n}^{\circ} 1 \mid$ Automne 2015

«Une fabrique collective du patrimoine littéraire (XIX ${ }^{\mathrm{e}}-\mathrm{XXI}^{\mathrm{e}}$ siècles). Les collections de monographies illustrées » 
une sorte de "Racine du $\mathrm{xx}^{\mathrm{e}}$ siècle en proie à une époque sans génie ", pris dans « le temps creux de l'histoire, où le lien qui unit des valeurs spirituelles en plein éclat et des valeurs de civilisation en pleine décadence ${ }^{40} »$. De ce point de vue, si toute la vie de Giraudoux est à considérer, ses actions politiques de la fin des années 1930 deviennent l'épisode clé de ce parcours intellectuel afin, comme l'avance André Bazin, de ne pas «manqu[er] l'heure grave où Giraudoux, sur le tard, déchanta, vieillit très vite et mourut ${ }^{41}$ ».

Ce «désenchantement» de Giraudoux, dont son parcours politique témoigne, est au cœur du travail de réhabilitation de Chris Marker. Les engagements de Jean Giraudoux sont complexes et font l'objet d'interprétations très diverses. Comme d'autres, cet écrivain suscite des célébrations admiratives et des dénonciations qui brouillent les analyses historiques. Plusieurs éléments de son parcours nourrissent des lectures contradictoires. À commencer par sa condition d'«oblat de la $\mathrm{III}^{\mathrm{e}}$ République $^{42}$ », lui qui devient diplomate et critique du régime parlementaire, après avoir été décoré pour ses activités militaires durant la Première Guerre. Sa carrière de romancier se double, à compter de l'adaptation de Siegfried, d'une position d'auteur majeur du théâtre de son temps. Ce " génie fantaisiste ${ }^{43}$ », doté d'une large audience, est un talentueux manipulateur des ressorts littéraires du rêve et des mythes qui lui permettent de traiter des questions sociales. Les proximités modernisatrices de Giraudoux avec les milieux technocratiques à la recherche d'une «troisième voie » dans les années 1930 - à mettre en perspective avec sa connaissance profonde de l'Allemagne ${ }^{44}-$, mériteraient d'être approfondies. Comme l'écrit Pierre Vidal-Naquet, à la veille d'une guerre certaine, Giraudoux, avec son livre Pleins pouvoirs, "n'envisage [...] aucunement un combat de la démocratie contre l'État raciste et national-socialiste d'Hitler. Il est un homme qui exprime son appréhension devant la décadence de la nation qui est la sienne. » Depuis cette perspective, la question de la «race française », c'est-à-dire d'une politique raciale dont l'État doit se saisir, devient pour l'écrivain-diplomate le problème essentiel ${ }^{45}$. Les conférences compilées dans Pleins pouvoirs sont aussi l'occasion pour Giraudoux de faire des propositions concernant l'urbanisme - en 1928, il a fondé «La Ligue urbaine»-, l'hygiène ou la baisse des naissances. S'il n'est pas le seul à les défendre à cette époque, ses idées, notamment celles qui dénoncent brutalement l'immigration, sont portées par une notoriété considérable, une écriture

Vol. 7, $\mathrm{n}^{\circ} 1 \mid$ Automne 2015

«Une fabrique collective du patrimoine littéraire (XIX ${ }^{\mathrm{e}}-\mathrm{XXI}^{\mathrm{e}}$ siècles). Les collections de 
puissante et l'exigence d'une certaine modernité technique. La nomination de Jean Giraudoux à la tête d'un Commissariat général à l'information et à la propagande en juillet 1939 répond à la volonté d'action de l'écrivain. Cette responsabilité, au service d'une "union sacrée», sera exercée dans une conjoncture difficile, rendue plus compliquée encore par la précarité institutionnelle du Commissariat, pris dans l'exacerbation des concurrences administratives et politiques qui rendra la position acceptée par Giraudoux rapidement «intenable ${ }^{46}$ ». En mars 1940, après la chute du gouvernement d'Édouard Daladier, Giraudoux quitte ses fonctions. Il se consacre alors notamment à l'écriture pour le cinéma et le théâtre, rédige Sans pouvoirs en écho à son essai de 1939, et s'intéresse toujours à l'urbanisme en signant, par exemple, la préface de la première édition de La Charte d'Athènes de Le Corbusier (1943), avec qui il partageait de nombreuses préoccupations ${ }^{47}$. Durant l'Occupation, puis après sa mort en janvier 1944, et donc dès la fin de la guerre, son œuvre est l'objet de revendications opposées.

\section{Des pleins pouvoirs de l'imagination au service de la politique}

Les places de Pleins pouvoirs et de la direction du Commissariat à l'information sont pleinement expliquées par Marker, qui y revient à plusieurs reprises tout au long de son essai. Selon lui, Giraudoux pense « le pouvoir politique [comme] un moyen d'incarner en son pays les valeurs de ses romans ». Il s'agirait d'une position originale, jamais incarnée auparavant: lorsque les deux «domaines» correspondent, à l'instar de Malraux, c'est dans un rapport inverse que la correspondance est établie, de la politique vers la littérature. Si Giraudoux «a consacré tant de temps à disposer dans ses manuscrits les maquettes d'un monde viable, c'est qu'il comptait les soumettre aux entrepreneurs de ce monde-ci ». Il s'agit bien de présenter le Giraudoux de 1938 comme un auteur engagé et précurseur de ce point de vue. Sartre? À cette date, il «n'a pas encore accroché le grelot de la littérature engagée [...].». Gide? Ses «aventures » avec l'URSS interrogent sa conception du «devoir de l'artiste ${ }^{48} »$. Selon Marker, la singularité giralducienne provient du fait que « [c]'est son action qui témoigne de son œuvre ». Ayant signalé l'intérêt de l'écrivain pour l'urbanisme et le sport, il évoque une «imperturbable décalcomanie de l'œuvre sur la vie». Et c'est bien le monde des corruptions, des ambitions, des angoisses et des peurs,

Vol. 7, $\mathrm{n}^{\circ} 1 \mid$ Automne 2015

«Une fabrique collective du patrimoine littéraire (XIX ${ }^{\mathrm{e}}-\mathrm{XXI}^{\mathrm{e}}$ siècles). Les collections de monographies illustrées » 
celles de la III ${ }^{e}$ République accaparée par « des avocats, des vieillards et des hommes d'argent», qui explique que cet homme dont la maitrise de l'imagination était la seule arme voulait devenir responsable politique afin, pensait-il, de refaire la France selon ses valeurs. Il ne comprenait alors pas, dans un contexte qui "venait de changer du fait des événements", que le «Ministère de l'Imagination, qu'il avait réclamé, était devenu le Ministère du Mensonge ${ }^{49} »$.

Où en serait Giraudoux aux débuts des années 1950? Son «obsession de la France seule », qui est «le petit choc qui brise le charme» de Pleins powvoirs, c'est-à-dire «cette méconnaissance de la répartition des forces d'asservissement et des forces de libération dans le monde », l'aurait-elle entraîné vers «les défenseurs des valeurs spirituelles de l'Occident [...] »? Non, répond Marker. Toutefois, son rôle au Commissariat à l'Information demeure le moment ineffaçable ${ }^{50}$. Depuis cette «seule faute», faire de Giraudoux un «fasciste » est cependant «absurde ». L'écrivain s'est perdu, car « le langage est le produit d'une société ». Lui qui incarnait les «valeurs absolues de civilisation» s'est pris au jeu de l'action qui caractérisait les années d'avant-guerre et il a mis «le langage avant les hommes ${ }^{51}$ ».

Le fil de la démonstration devrait être détaillé, pour reconstruire, par exemple, le parallèle avec Racine, historiographe du Roi qui a occupé Giraudoux, les subtilités mobilisées par Marker pour définir le réalisme giralducien et son sens de l'allégorie qui le rapproche du Moyen Âge, ou encore les articulations entre les textes et les éclairages biographiques qui accompagnent la partie anthologique. Retenons ici qu'il s'agit bien d'inscrire Giraudoux dans les débats de son temps, de lui (re)donner une légitimité52. Pour Marker, l'œuvre de Giraudoux est un recours pour lier sa conception d'un art « régénérateur ${ }^{53}$ » et le devenir de la Cité, particulièrement face aux formes de l'engagement représentées par celui qui occupe alors tout l'espace intellectuel, ou presque: Jean-Paul Sartre ${ }^{54}$. Cette présence sartrienne est l'un des motifs de l'essai de Chris Marker. Récurrente, par rapport aux autres références mobilisées (Aragon, Gide, Cocteau, Montherlant...), elle suscite même des doutes chez un critique, bienveillant par ailleurs. En effet, Guy Dumur s'interroge sur l'instrumentalisation de cette opposition, car il estime que Sartre est plutôt un allié de Giraudoux ${ }^{55}$.

Vol. 7, $\mathrm{n}^{\circ} 1 \mid$ Automne 2015 
Immense lecteur, notamment lors des années passées à l'École normale, Sartre avait lu Giraudoux avec attention ${ }^{56}$. Celui-ci est même un modèle, car il s'est éloigné avec réussite du professorat, destin traditionnel des normaliens, pour devenir un écrivain reconnu. En effet, Sartre partage avec Giraudoux le fait d'être un représentant de «la conversion réussie de normalien en écrivain » qui devient plus fréquente dans l'entre-deux-guerres, signe "d'une transformation en acte des relations entre universitaires et écrivains ${ }^{57}$ ». Parmi ceux qui franchissent alors ce cap - dont Romain Rolland, Paul Nizan ou Jules Romains, s'éloignant ainsi d'un modèle plus strictement «littéraire » incarné par André Gide -, Giraudoux est celui qui parvient à la plus grande notoriété.

Parmi les moments où la position nouvelle et dominante qui sera celle de Sartre dans les années d'après-guerre se forge, on peut mentionner une série de critiques qu'il donne, notamment, à $L a N R F$. Ces textes concernent des maitres que Sartre revendique, des écrivains de son âge auxquels il se mesure et encore des aînés, dont Jean Giraudoux ${ }^{58}$. En mars 1940, son article sur Choix des élues (paru au printemps 1939) est l'occasion de mettre en place sa lecture philosophique du romanesque (qu'il réalise dans ses romans après la guerre). Selon lui, et Marker cite ce jugement à la page 17 de son essai, la littérature de Giraudoux relève d'un rapport «schizophrène » au réel. C'est un espace à part entière et différent que l'écrivain a construit avec sa plume. Sartre décrit les « formes substantielles », les « archétypes » de ce «monde en ordre » dont la seule dynamique possible relève des mouvements de formes figées. En effet, on n'y trouve aucun déterminisme, aucune contingence, ni attention forte aux commencements, aux «virginités reconquises", à la réalisation de l'essence des êtres et des choses que porte le temps d'un «éternel matin» du recommencement. "L'univers de M. Giraudoux est une Histoire naturelle. » La place de l'Homme y est donc limitée, poursuit Sartre, par un système de classement quasi immuable et l'écrivain «n'est rien d'autre qu'un employé du cadastre ». Lui-même surpris par cette filiation, Sartre renvoie l'inspiration de Giraudoux à la pensée d'Aristote. Alors que les philosophes et les savants pensent le devenir et la liberté du jugement depuis quatre siècles, la morale giralducienne est celle d'une soumission «à la nécessité des archétypes ». Pourquoi Giraudoux recourt-il à ces archétypes pour bâtir un monde? Sartre dit l'ignorer, mais peut-être que cet écrivain «si discret et qui s'efface devant ses fictions nous parlera-t-il un jour de lui ${ }^{59} »$.

Vol. 7, $\mathrm{n}^{\circ} 1 \mid$ Automne 2015

«Une fabrique collective du patrimoine littéraire (XIX ${ }^{\mathrm{e}}-\mathrm{XXI}^{\mathrm{e}}$ siècles). Les collections de monographies illustrées » 
Cette analyse n'a pas la vigueur de celle que Sartre a consacrée à La Fin de la nuit de François Mauriac (La NRF, février 1939). Elle est cependant tout aussi brillante et partage certaines interrogations, philosophiquement armées, sur l'art romanesque et la liberté. Chris Marker tente d'y répondre dans son essai. Il amorce sa défense par la question du refus du réel posée par Sartre. Reprenant des exemples que ce dernier utilisait pour frapper l'esprit de ses lecteurs, il reconnait : « on s'interroge en effet sur le sens de ce monde parfait, verni, où tout arrive pour la première fois, où l'archétype de la femme mange un archétype de cornichon». Marker construit son argumentation sur une analogie avec les moyens que se donne le cinéma américain pour faire exister les actions par l'image. Et c'est une autre dimension de l'interpellation qui s'ouvre ici puisque Sartre, on le sait, est très attiré par l'art cinématographique. De fin 1943 à fin 1945, il a été embauché par Pathé-Cinéma pour écrire des scénarios, ceci sous la direction de Jean Giraudoux qui était, lui aussi, employé par le producteur. En 1945, lors d'un séjour aux États-Unis, l'écrivain-philosophe rédige une série d'articles remarqués sur la production cinématographique américaine. Marker mobilise l'exemple de Citizen Kane d'Orson Welles, auquel Sartre reprochera son intellectualisme à l'occasion d'une critique qu'il regrettera par la suite ${ }^{60}$. Chez Welles, comme dans l'écriture giralducienne, «le décor devient l'action ", autrement dit, le but suprême de " cet étrange romancier n'est pas la conduite d'une action à laquelle on prétendrait scélératement nous faire croire, mais la construction d'un univers dont l'existence est le seul enjeu du livre». Il s'agit bien de rompre avec la réalité et de construire un rapport direct au lecteur. La capacité de Giraudoux de mener un «vrai roman » est attestée aussi bien par Combat pour l'ange que Choix des élues, précise Marker, et s'ils sont apparemment irréalistes c'est parce qu'ils veulent donner «une image du bonheur humain ». En cela, se méfiant des romanciers qui trichent en jouant sur «l'équivoque habilement entretenue d'un monde sur mesures et le monde démesuré », il est convaincu que Giraudoux se situe du côté de "l'honnêteté »: il instaure un dialogue vrai avec le lecteur et il ne tient qu'à celui-ci de s'en saisir. L'essence de cet art est l'allégorie et, de ce point de vue, Giraudoux relève d'une inspiration médiévale, déjà repérée par Aragon, Claude Roy ou Jean Prévost. Cette manière de connaitre par l'Allégorie n'est pas fausse comme Sartre le prétend en dénonçant des «intuitions improductives » qu'il replie sur une « illusion de la fausse reconnaissance »,

Vol. 7, $\mathrm{n}^{\circ} 1 \mid$ Automne 2015

«Une fabrique collective du patrimoine littéraire (XIX ${ }^{\mathrm{e}}-\mathrm{XXI}^{\mathrm{e}}$ siècles). Les collections de 
définie par la psychologie. L'existentialisme sartrien justifie probablement ce parti-pris, mais la perspective essentialiste de Giraudoux est légitime. Par elle, celui-ci a «enraciné l'au-delà dans l'immanent», et grâce à cela, reprenant encore les exemples de la démonstration sartrienne, «il n'y a plus d'écart entre l'être et son apparence [...] ce n'est pas dans une déchirure sur un autre monde, mais dans l'intimité des rues et des cornichons que se glisse la révélation ${ }^{61} »$.

Poursuivant une démonstration très pointue, en convoquant le cinéma italien néo-réaliste puis français, avec Marcel Carné et Prévert, c'est un réalisme giralducien que Marker met en avant, qu'aucune reconstitution réaliste ne peut reproduire. L'œuvre de Giraudoux relève certes de la féérie, mais son point de départ est "une esthétique de la vérité ». Les détails réalistes omniprésents dans les romans de Jean-Paul Sartre - est évoqué "Drôle d'amitié », un extrait des Chemins de la liberté - sont autant de vues de l'esprit. Par un renversement impossible à détailler ici, Marker oppose la vérité de «l'épopée » d'Aragon, avec Les Communistes (1949-1951), aux faux communistes de la prose sartrienne (il pense à La Mort dans l'âme, troisième tome des Chemins de la liberté (1949)). Cette revendication positive du roman à thèse d'Aragon intervient à un moment où celui-ci met tout son art et son prestige au service du Parti communiste ${ }^{62}$. Plus largement, et Marker revient sur ses conceptions d'une esthétique du roman moderne: le «romanromanesque » est un piège et les attaques contre Giraudoux au nom de son soi-disant réalisme ou irréalisme sont absurdes. En effet, la forme romanesque est «la plus réussie des entreprises de diversion que l'individualisme bourgeois ait opposée au monde de la fraternité et de la solidarité [...] ». Ceci dit, selon Marker, Giraudoux apparaît bien comme le seul écrivain «à chercher une solution au problème de la liaison du romancier avec le monde ». Que celle-ci soit fragile ou fausse, c'est possible, mais elle est avancée. Avec l'idée de "séparation », qu'il reprend d'un article qu'il a publié dans Esprit en 194763, Marker entend signifier que le roman contemporain doit être un "déracinement absolu», un "dépaysement absolu » où «l'univers romanesque trouve sa profondeur dans ce décalage de son monde avec le nôtre, ce décadrage qui donne leur relief aux images du stéréoscope, et dont le héros est précisément la mesure ${ }^{64} \gg$. La forme romanesque relève en fait du conte et le roman est même «la version censurée par la raison du conte de fées. Alors que la féerie de Giraudoux est

Vol. 7, $\mathrm{n}^{\circ} 1 \mid$ Automne 2015

«Une fabrique collective du patrimoine littéraire (XIX ${ }^{\mathrm{e}}-\mathrm{XXI}^{\mathrm{e}}$ siècles). Les collections de monographies illustrées » 
la version censurée par la politesse, la légèreté et la pudeur, d'une très sérieuse approche du monde.»Et, afin de boucler son raisonnement, Marker affirme que l'action politique de l'écrivain devenu propagandiste d'État est bien produite par les valeurs de ses romans ${ }^{65}$. À la lecture de ce Giraudoux par lui-même qui porte une réhabilitation au prix d'une théorie du réalisme romanesque - une réflexion que Marker poursuivra, sur d'autres bases, avec son travail sur les images, car il dit douter de l'intégrité du roman et du théâtre ${ }^{66}$-, on perçoit des effervescences littéraires dont Esprit, le Seuil ou Peuple et Culture furent les supports, avec, par exemple, la parution remarquée de Je vivrai l'amour des autres de Jean Cayrol au Seuil en 1947. La réception par la critique de l'essai de Marker montre la manière singulière dont sa lecture de Giraudoux est alors perçue.

\section{Une héroïsation discutée}

Selon différentes modalités, les critiques réagissent à la volonté de Marker de faire de l'œuvre de Jean Giraudoux l'étendard d'une nouvelle expression littéraire des années incertaines de l'après-guerre. La vivacité des conceptions défendues, le débat engagé avec l'existentialisme de Sartre, le début de reconnaissance obtenu par Marker avec son roman, Le Cour net, paru en 1949, ne suscitent toutefois qu'une attention relative et l'espace de réception de l'édition de 1952 est en effet circonscrit ${ }^{67}$. Si on laisse de côté les brèves mentions, souvent liées à la série dans son ensemble, la moitié des articles de fond - nous en avons repéré six - sont le fait de proches du Seuil, d'Esprit et de Marker. Deux revues catholiques - généralement attentives à la production du Seuil -, Études et La Vie intellectuelle, apparaissent dans cette liste. La Table Ronde est le périodique le plus singulier de cette réception. Cette revue est alors entrée dans un moment de transition : fondée en 1948 autour de Thierry Maulnier et François Mauriac par la maison d'édition éponyme pour concurrencer Les Temps Modernes de Sartre, l'ensemble est racheté par Plon en 1950. Dès lors, des changements internes conduisent Mauriac à s'en éloigner en 1953, puis à faire de la revue un point d'ancrage des écrivains du groupe des «Hussards ${ }^{68}$ ». Les spécificités des «Écrivains de toujours », dont le lancement est récent, et le fait que le Seuil soit encore une entreprise modeste, identifiée au pôle catholique du monde intellectuel et dont les capacités promotionnelles sont réduites, jouent sans aucun doute un rôle dans la reconnaissance limitée dont ce livre est l'objet. Sur le fond,

Vol. 7, $\mathrm{n}^{\circ} 1 \mid$ Automne 2015

«Une fabrique collective du patrimoine littéraire (XIX ${ }^{\mathrm{e}}-\mathrm{XXI}^{\mathrm{e}}$ siècles). Les collections de 
les critiques repèrent parfaitement les ambitions de Marker et se focalisent sur la question de l'utilité à la fois politique et esthétique de l'œuvre de Giraudoux aux débuts des années $1950^{69}$.

Même chez ses plus proches alliés, la démarche de Marker suscite des réserves que les enthousiasmes n'éteignent pas. Claude-Edmonde Magny, essayiste, collaboratrice d'Esprit et du Seuil, a consacré en 1945 un livre à la rhétorique du «magicien Giraudoux » - sur laquelle Marker prend largement appui dans son livre ${ }^{70}$. Pour une part, l'étude de Magny fait aussi écho à l'article de Sartre et s'arme de la pensée philosophique pour mettre en avant «la passion du superlatif» de Giraudoux, dont elle reconnait, sans qu'elle trouve justifié d'en faire un défaut, que l'art est teinté des « faiblesses d'une littérature d'évasion ». Elle exige moins de l'écrivain sur le terrain de son engagement, mais son livre participe d'une même volonté de le faire exister sur la scène littéraire contemporaine. Ainsi, de cette préciosité qu'elle étudie au fil des textes, elle pointe une préoccupation partagée par d'autres auteurs, dont Maurice Blanchot, critique à la notoriété forte après la guerre ${ }^{71}$. À l'occasion d'un bref compte rendu dans un quotidien généraliste, Magny encense la lecture biographique de Marker, mais elle demeure en retrait par rapport aux affirmations de ce dernier: elle estime que le «mystère » de l'écrivain reste intact. Pour elle, la "permanence de Giraudoux » est acquise et la compréhension de ses engagements reste dépendante d'une "part d'ombre de plus en plus intégrée à cette œuvre si radieuse ${ }^{72} »$. Ce double mouvement d'enthousiasme et de scepticisme est encore perceptible dans l'article qu'Albert Béguin donne aux pages littéraires de La Gaz̨ette de Lausanne. Celui-ci est une grande figure de la Résistance littéraire et de la critique, probablement l'auteur le plus reconnu de ce point de vue à fournir en textes la revue Esprit. Depuis 1947, il est aussi une recrue très importante des éditions du Seuil où, comme éditeur, il publie ses découvertes littéraires, dont Jean Cayrol. De plus, il a été le premier animateur de la série «Écrivains de toujours» avant de prendre la direction de la revue personnaliste après la mort brutale en 1950 de son fondateur, Emmanuel Mounier. Dans son papier pour le quotidien suisse, Béguin salue avec force la performance toute giralducienne de Marker. Ce livre lui parait remarquable autant pour le choix des textes que pour l'implication déployée pour revendiquer un tel héritage. Si la méthode de Marker, très concernée par la vie et l'œuvre dépeintes, inspire quelques critiques à Béguin, elle

Vol. 7, $\mathrm{n}^{\circ} 1 \mid$ Automne 2015

«Une fabrique collective du patrimoine littéraire (XIX ${ }^{\mathrm{e}}-\mathrm{XXI}^{\mathrm{e}}$ siècles). Les collections de monographies illustrées » 
ressuscite chez lui les sensations liées à sa découverte de Giraudoux, « jusqu’à la légère déconvenue » provoquée par «l'optimisme [...] un peu factice » des textes d'actualité publiés par celui-ci à la veille de la guerre. Et Béguin, en contrepoint (amical) à la vision de Marker, de mobiliser ses propres souvenirs de Giraudoux, dépeint comme toujours en éveil et discret dans les années 1930, puis, durant la guerre, « désemparé » face à un monde «cruel». Il fait un pas de plus que Claude-Edmonde Magny quant à la certitude de Marker de voir en Giraudoux un recours pour l'époque contemporaine, car il estime que cette conscience déchirée, qui s'est éloignée de sa volonté de contribuer avec sa plume à changer le monde en paradis, subsiste aujourd'hui comme une «valeur durable » qui exprime «les espoirs et les désespoirs de ce demi-siècle mieux que des voix plus sonores aujourd'hui ${ }^{73} »$. Spécialiste du romantisme allemand, observateur avisé de l'Allemagne où s'installe le nazisme, converti au catholicisme en 1940, éditeur de la Résistance, Béguin pensait lui aussi la littérature comme un engagement. Il laisse toutefois de côté, au moins d'une manière explicite, le dialogue engagé par Marker avec Sartre. C'est dans Esprit, sous la plume d'André Bazin, que paraît la recension dont l'influence a pu être la plus importante pour la diffusion du livre; un lieu où Marker s'exprime beaucoup, comme nous l'avons dit, et une critique qui émane d'un proche lui aussi engagé au sein du mouvement Peuple et culture. Bazin se situe dans une note similaire à celle de Béguin, en insistant positivement sur l'identification giralducienne de Marker et le parti pris de ne pas esquiver les «déficiences» de son héros. Et Bazin, qui eut l'occasion de rencontrer Giraudoux en 1943, conclut son texte en évoquant le refus des facilités d'une posture d'homme de lettres que celui-ci affiche alors pour rester, malgré tout, «uniquement écrivain, afin d'être mieux homme ${ }^{74}$ ». De ces recensions émanant d'un cercle de proches, on peut retenir l'évocation commune de la permanence giralducienne, soutenue par la continuité de cette personnalité par-delà ses engagements politiques, et, surtout, le décalage de ses ambitions face à des événements qu'il n'aurait pas véritablement saisis. Le projet passionné de Marker de faire de Giraudoux la référence de l'engagement littéraire dans les années 1950 n'est donc qu'indirectement discuté.

Si l'on élargit le cercle, on remarque que le point de départ de la critique de Claude Roy est à nouveau le constat de l'appartenance de Marker à la

Vol. 7, $\mathrm{n}^{\circ} 1 \mid$ Automne 2015

«Une fabrique collective du patrimoine littéraire (XIX ${ }^{\mathrm{e}}-\mathrm{XXI}^{\mathrm{e}}$ siècles). Les collections de 
"famille Giraudoux, comme Giraudoux faisait partie de la famille La Fontaine ». Selon lui, deux informations principales émanent de cet essai : Giraudoux est bien quelqu'un de «délicieusement important»; et son brillant biographe «a toutes les chances et les dons d'être, lui aussi, important ». Le cadet n'est pas dupe des difficultés de Giraudoux qui a trop cru au «langage comme exorcisme et pas assez à la politique comme solution ». Et Roy, qui durant la guerre a quitté les milieux maurrassiens où il était engagé pour la Résistance avant de devenir un journaliste reconnu, admet, à quelques réserves près, l'idée d'un Giraudoux pris dans les enjeux de la société de son temps, et décrit comme une sorte de «sorcier» à deux faces, l'une admirable, l'autre impossible. Mais il souligne qu'il faut remercier Marker pour son rappel que «la France de Giraudoux est plus ressemblante que celle des politiciens », et de lui avoir élevé une "stèle qui est en ellemême un chef-d'œuvre d'intelligence et d'élégance, la seule qui importe, celle du cœur ${ }^{75} »$.

$\mathrm{Si}$ «l'homme» Giraudoux demeure pour les proches d'Esprit la qualité à retenir, et que Claude Roy mise sur l'intelligence du cœur et donc des sentiments, c'est dans un registre d'abord politique puis moral qu'André Séailles, dans $\mathrm{La}$ Table Ronde, va situer ses jugements. Pour commencer, il reconnaît que rendre hommage à Giraudoux est une entreprise difficile, car elle implique de contourner la limite de Giraudoux, cette ignorance aristocratique d'un monde, le nôtre, où règnent le mal et la souffrance ». Le critique retient que la défense de Marker entend démontrer que son auteur n'est en rien "réactionnaire », aussi bien littérairement que politiquement. Giraudoux ne serait donc pas le «rêveur indifférent aux problèmes de son temps ", et Marker, s'il commet l'erreur d'imaginer ce que l'écrivain aurait pensé de certains événements contemporains (comme la Guerre froide et le réarmement allemand) insiste à raison sur ce point. Pour André Séailles aussi, Giraudoux s'est, avant Sartre, interrogé sur la place sociale de l'écrivain. Il concevait que la Cité devait mettre «son appareil technique au service de l'imagination créatrice». Et c'est ainsi qu'il faut comprendre ses prises de position sur l'urbanisme ou le sport. Les «terribles lacunes» de Giraudoux n'en sont pas moins évidentes durant les mois qui précédent la Deuxième Guerre mondiale. Penser que la civilisation pouvait se défendre contre la violence qui se déchaînait alors par le seul rempart de ses valeurs spirituelles fut une erreur. Selon Séailles, Giraudoux est un écrivain engagé

Vol. 7, $\mathrm{n}^{\circ} 1 \mid$ Automne 2015

«Une fabrique collective du patrimoine littéraire (XIX ${ }^{\mathrm{e}}-\mathrm{XXI}^{\mathrm{e}}$ siècles). Les collections de monographies illustrées » 
quand il ne joue pas «les augures ». Marker est très convaincant quand il affirme que «c'est sur le plan de l'éternité [qu'il] est le plus actuel». De Giraudoux, il faut donc retenir l'optimisme et le bonheur dont la source est la fraternité; son œuvre est traversée des conflits de l'amour et d'une «tendresse fraternelle [qui] se heurte parfois durement aux angles de la réalité ${ }^{76} »$.

Ce sont d'abord les erreurs de Giraudoux, et le «cinglant démenti » que l'Histoire lui a infligé, que mobilise Jean Mauduit, dans Témoignage chrétien. «L'éternelle aurore » imaginée par l'écrivain s'est évanouie. Son œuvre est aujourd'hui méconnue par les moins de 20 ans et les écrivains engagés, à l'instar "des Sartre des deux sexes», vouent un mépris certain à cet « incorrigible esthète ». Néanmoins, le mérite de Marker est de montrer que le dédain présumé de Giraudoux pour les « réalités de son temps » est une vision fausse. Car il donnait à sa littérature la mission de «bâtir l'univers ». Mais, selon Mauduit qui défend une vision très classique de la littérature, Marker s'éloigne de cette réalité lorsqu'il peint un écrivain trop confiant dans les pouvoirs du langage, alors que celui-ci est le reflet de l'état d'une société. Et ici le critique récuse l'assimilation de la parole politique et de la parole littéraire : la «grandeur» de Giraudoux est d'être resté un écrivain, " pour le meilleur et pour le pire », mais pour agir sur le monde. Et il serait paradoxal d'exempter Giraudoux de ses péchés d'esthétisme pour en faire un écrivain engagé. Marker s'égare, car «il n'y a pas d'écrivains communistes, ni fascistes, ni réactionnaires, mais de bons et de mauvais écrivains ${ }^{77} . . . ~ A u$ nom d'une autre revue catholique, La Vie intellectuelle, le chroniqueur refuse la volonté d'action de l'auteur de Pleins powvoirs. En effet, d'après Gabriel Venaissin, Giraudoux, "l'un des plus remarquables exemples de la technocratie littéraire », fut «celui qui a fait des héros à partir de l'époque qui en manqua le plus». Puis, «envoyé spécial d'un monde supérieur», il se trouva engagé dans des responsabilités triviales: «Ce fut dramatique. Ce fut navrant ${ }^{78}$. " Mais à ce pôle catholique, c'est Pierre de Boisdeffre qui exprime la réaction la plus négative dans la publication jésuite Études. Comme Claude Edmonde-Magny et son Précieux Giraudoux, Marker entend démontrer que cet écrivain «est un auteur antibourgeois, antipharisien, engagé, bien pensant - bien pensant de gauche évidemment. Pauvre Giraudoux! » Pour le critique (par ailleurs haut fonctionnaire), cette perspective qui dénature Giraudoux est heureusement démentie par les

Vol. 7, $\mathrm{n}^{\circ} 1 \mid$ Automne 2015

«Une fabrique collective du patrimoine littéraire (XIX ${ }^{\mathrm{e}}-\mathrm{XXI}^{\mathrm{e}}$ siècles). Les collections de 
textes choisis. Et quoi que fasse Christian Marker, Giraudoux n'est pas « un Sartre avant la lettre » comme il voudrait le montrer, mais demeure «le plus léger, le plus gracieux, le plus délié, le plus subtil de nos lettrés, notre meilleur écrivain chinois ${ }^{79} \gg$.

L'ensemble de ces recensions montre un écart relatif entre le projet de Marker d'une littérature engagée dont le modèle giralducien serait la première expression, et sa perception au moment de la sortie de l'essai. Audelà du nombre restreint des critiques, leur concentration autour de deux pôles proches (et dominés littérairement) des revues intellectuelles, indique que l'ambition de Marker ne fait écho qu'à des préoccupations limitées dans l'espace intellectuel de l'époque. Significativement, la seule revue littéraire qui prend en charge son essai est $\mathrm{La}$ Table ronde. La réflexion autobiographique que Christian Marker mène avec ce livre sur Giraudoux, l'effacement partiel de cette œuvre, les positions conjointement subalternes d'Esprit et du Seuil ${ }^{80}$, ou encore l'omniprésence littéraire, philosophique et militante de Jean-Paul Sartre et de sa revue Les Temps modernes, contribuent à limiter l'entreprise de réhabilitation tentée par Marker. La mise en avant de «l'homme» Giraudoux, de sa personnalité, de la fraternité qu'elle incarnerait, et, en quelque sorte, cette dépolitisation par une perception esthétique qui devient première, car elle guiderait l'action, participent bien de l'affrontement qui se joue alors entre l'existentialisme sartrien et le personnalisme d'Emmanuel Mounier, dont la revue Esprit est le fer de lance. Cette lecture de Giraudoux intervient à un moment où les conséquences de la guerre imposent au monde intellectuel français un régime de surpolitisation. La dépolitisation esthétique de Jean Giraudoux, qui demeure ancrée dans un outillage « littéraire » face à une vision " philosophique » plus large qu'impose alors Sartre, est un coup de force qui ne peut rallier largement les suffrages et qui joue encore comme un frein au retour en grâce de cet écrivain que souhaitait Marker.

De ces entrecroisements entre ressources biographiques, revendications et réappropriations d'un héritage et modalités d'une consécration littéraire, il faut aussi retenir que Chris Marker poursuit son investissement giralducien à terme. Alors même qu'il s'implique résolument à compter du milieu des années 1950 dans les mondes audiovisuels et que cette nouvelle phase de sa carrière intellectuelle est construite en rupture avec la précédente. Après

Vol. 7, $\mathrm{n}^{\circ} 1 \mid$ Automne 2015

«Une fabrique collective du patrimoine littéraire (XIX ${ }^{\mathrm{e}}-\mathrm{XXI}^{\mathrm{e}}$ siècles). Les collections de monographies illustrées » 
celle de 1952, les éditions successives du Giraudoux par lui-même en 1959, 1962 et 1978 permettent de repérer des modifications du texte dont l'amplitude demeure, toutefois, assez faible. De même que le texte, les illustrations sont revues à la marge, mais toujours dans le sens d'une inscription de Giraudoux dans l'actualité 81 . Toutefois, ces reprises révèlent l'investissement particulier de Marker dans la défense de son auteur fétiche. En effet, ce suivi est une exception si l'on considère la distance que Marker a pu mettre avec ses productions éditoriales et livresques des années 1940 et 1950. Et, dans les années 1990 encore, il répond avec attention à la demande du Seuil pour une nouvelle révision de son essai dans le cadre d'une possible relance de la série «Écrivains de toujours ${ }^{82}$ ». Cette attention à Giraudoux s'exprime encore par-delà le livre paru en 1952, tout particulièrement aux débuts des années 1960, au moment où le cinéma devient la préoccupation majeure de Marker. Ainsi, la première partie du film Le Joli mai, réalisé en 1962 par Pierre Lhomme et Chris Marker, débute sur une longue citation de Giraudoux sur Paris. À l'occasion de l'un de ses rares entretiens, toujours en cette année charnière 1962, Marker affirme: "Jean Giraudoux est le plus grand écrivain engagé de notre époque, on s'en rendra peut-être compte un jour ${ }^{83}$.»

Durant ces quelques années riches en productions diverses, pour certaines méconnues, Marker s'exprime d'abord comme un écrivain et un essayiste. Avec conviction, il entreprend de replacer Giraudoux sur l'échiquier littéraire. Faisant le lien entre sa propre socialisation artistique et politique, et les questionnements qui sont les siens durant ces années d'après-guerre - où sa propre reconnaissance est encore incertaine-, le coup de force giralducien est aussi porté par la volonté de lutter contre l'hégémonie intellectuelle de Jean-Paul Sartre. De ce point de vue, les proximités de Marker avec Esprit, qui oppose un existentialisme chrétien et, plus encore, le personnalisme de Mounier à Sartre, et celles avec le Seuil - qui récuse les positions du milieu des Temps Modernes ${ }^{84}$-, renforcent son entreprise. On sait que la puissante appropriation sartrienne de l'engagement et de la responsabilité de l'écrivain, particulièrement défendue au pôle conservateur du champ intellectuel, est autant combattue par les communistes que par les «hussards» de La Table Ronde - dans le contexte des débats sur l'épuration ${ }^{85}$, que clôt en 1952 la seconde loi d'amnistie. Dans cette conjoncture singulière, la lecture proposée par Marker des années d'après-

Vol. 7, $\mathrm{n}^{\circ} 1 \mid$ Automne 2015

«Une fabrique collective du patrimoine littéraire (XIX ${ }^{\mathrm{e}}-\mathrm{XXI}^{\mathrm{e}}$ siècles). Les collections de monographies illustrées » 
guerre au prisme d'un usage de l'imaginaire empruntée à Giraudoux est une position inédite. Certes, elle demeure marginale, ce dont témoigne la faible réception critique de l'essai. L'investissement de Marker pour une nouvelle consécration de Jean Giraudoux constitue l'ultime étape des expérimentations d'une nouvelle expression esthétique, dont le cinéma sera dorénavant le vecteur privilégié.

Hervé Serry, sociologue, est directeur de recherche au Cnrs, au sein de l'UMR Cresppa dont il est directeur. Ses travaux et ses enseignements portent sur les mutations de l'édition contemporaine. Il est professeur associé de l'Université de Sherbrooke. Parmi ses publications: «A Publishing Decision under Constraint. Samuel Beckett and Le Seuil Publishers in 1947 » (Journal of Beckett Studies, 2012); avec Josée Vincent, «Penser le rôle des foires internationales dans la mondialisation de l'édition. L'exemple des éditeurs québécois à la Buchmesse de Francfort » (Le Mouvement social, $\mathrm{n}^{\circ}$ 243, 2013/2); et en 2015, une monographie intitulée Aux Origines des éditions du Seuil (Paris, Seuil).

\section{Notes}

${ }^{1}$ André Bazin, à l'occasion d'une recension de la première édition de ce Giraudoux par hiimême, note l'usage du prénom Christian pour ce volume, alors que «Chris » est devenu la règle : «Malgré le prénom accru d'une syllabe, c'est bien notre Chris Marker qui a écrit ce petit livre parfait. »; André Bazin, Esprit, juillet 1952, p. 162-163.

${ }^{2}$ Je tiens tout particulièrement à remercier les deux relecteurs ou relectrices que le comité de la revue a désigné.e.s. Les remarques et conseils dispensés furent bienveillants et surtout très utiles. Également Cécile Delbecchi, assistante à la direction de Memoires du livre, dont les suggestions ont été précieuses.

3 Pour une présentation générale de la collection « Microcosme » inaugurée par «Écrivains de toujours»: Hervé Serry, "Une "vulgarisation de qualité" aux Éditions du Seuil. Conditions éditoriales pour la diffusion de nouvelles formes de savoirs (1945-1965) », L. Braida et B. Ouvry-Vial (dir.), Textes, Formes, Lectures en Europe (18 $\left.8^{\mathrm{e}}-21^{\mathrm{e}}\right), 2016$.

${ }^{4}$ Document sans titre, sans date [décembre 1949 - archives privées], reproduit dans Hervé Serry, Les Éditions du Seuil. 70 ans d'histoire, Paris, Seuil, 2008, p. 49.

5 Guy Dumur, "Souvenir de Giraudoux », Notre temps, 26 juillet 1952 (Archives des Éditions du Seuil, déposées à l'IMEC, Institut mémoire de l'édition contemporaine (Caen) - toutes les archives mobilisées pour cette recherche en relèvent). Du point de vue des publications consacrées à Giraudoux, durant la fin des années 1940 et le début des années 1950, l'activité critique et académique semble assez modeste, même si les publications d'un

Vol. 7, $\mathrm{n}^{\circ} 1 \mid$ Automne 2015

«Une fabrique collective du patrimoine littéraire (XIX ${ }^{\mathrm{e}}-\mathrm{XXI}^{\mathrm{e}}$ siècles). Les collections de 
inédit littéraire (Les contes d'un matin, Gallimard) et d'une conférence prononcée en Suisse en 1942 (Visitations, Grasset) procurent à Giraudoux une actualité éditoriale. Cf. Hasan Anamur, Bibliographie chronologique des publications de langue française sur Jean Giraudoux et son oeuvre de 1909 à 1970, Ankara, Université d'Ankara, 1980.

${ }^{6}$ Chris Marker, Jean Giraudoux in Selbstzengnissen und Bilddokumenten, Rowohlt, 1962. Un article paru dans L'École de la Nation en mars 1963 et signé «C. P.» signale que l'édition allemande est amputée d'une page : celle où Marker, après avoir évoqué Giraudoux pris dans «la vertu du verbe et de l'imagination», ne perçoit pas la réalité de la situation en Allemagne, dont les torches des célébrations nazies à Nuremberg qui symbolisent la montée définitive des violences. Pour l'édition française de 1962, p. 39.

${ }^{7}$ Christian Marker, Giraudoux par lui-même, Paris, Seuil, 1952, p. 5.

${ }^{8}$ Note, sans nom d'auteur, Modes de Paris, 17 août 1952.

9 René Lalou, «Feuilleton littéraire. Un inventaire de fictions », Hommes et mondes, août 1952, p. 590-591.

${ }^{10}$ Bamchade Pourvali, «Jeunesses de Chris Marker», Vertigo, 2013/2, n 46, p. 7-14. Voir aussi, sur un autre mode, Hervé Serry, "Chris Marker», http://www.seuil.com/pagehommage-chris-marker.htm [consulté le 20 juillet 2015].

${ }^{11}$ Lettre de Chris Marker à « Cher » [Paul Flamand], 9 septembre [1949].

12 David Martens, «L'exercice pseudonymique de l'autorité littéraire. Un partage des voix contesté », Emmanuel Bouju (dir.), L'autorité en littérature: exercice, partage, contestation, Rennes, PUR, 2010, p. 237-247.

${ }^{13}$ Chris Marker, Marker Mémoire, Paris, Cinémathèque française, janvier-février 1998. Les opportunités de recherche qu'ouvrent sans aucun doute le dépôt en 2013 des archives de Chris Marker à la Cinémathèque française par ses héritiers, et son inventaire en cours, permettront de mieux saisir ce parcours intellectuel, militant et artistique. Voir http://www.cinematheque.fr/fr/musee-collections/actualite-collections/dons-

acquisitions/archives-chris-marker-in.html [consulté le 17 juillet 2015].

${ }^{14}$ Cet hommage, auquel j'emprunte plusieurs éléments, apporte une brillante et très informée mise en perspective du parcours de Marker; François Lecointe, «Tombeau pour Chris Marker", Esprit, 18 octobre 2012. http://www.esprit.presse.fr/news/frontpage/news.php?code=232 [consulté le 17 juillet 2015].

${ }^{15}$ Simone Signoret, La Nostalgie n'est plus ce qu'elle était, Paris, Seuil, 1976, p. 32.

${ }^{16}$ Bernard Pingaud, Une tâche sans fin. Mémoires, Paris, Seuil, 2009, p. 92 sq.

${ }^{17}$ Extraits de «Nos Buts », La Revue française, 1er cahier, «Climats de France », juillet-août 1941. Voir la notice d'Olivier Cariguel, Panorama des revues littéraires sous l'Occupation, 19391945, Paris, Imec, 2007, p. 366-370.

Vol. 7, $\mathrm{n}^{\circ} 1 \mid$ Automne 2015

«Une fabrique collective du patrimoine littéraire (XIX ${ }^{\mathrm{e}}-\mathrm{XXI}^{\mathrm{e}}$ siècles). Les collections de 
${ }^{18}$ Bernard Pingaud, Inventaire, Paris, Gallimard, 1965, p. 15.

19 Période militaire et américaine notamment évoquée très brièvement par Chris Marker dans le film d'Agnès Varda, Agnès de ci de là Varda, 2011. Un témoignage littéraire signé d'un ami de Marker existe de cette époque; il est mentionné par François Lecointe, «Tombeau pour Chris Marker».

${ }^{20}$ Bernard Pingaud, Une tâche sans fin, Paris, Seuil, 2009, p. 93.

${ }^{21}$ Notice, «Chris Marker, Le Caur net roman».

${ }^{22}$ Dont le numéro «Les allemands parlent de l'Allemagne », Esprit, juin 1947.

${ }^{23}$ Hervé Serry, Aux Origines du Seuil, Paris, Seuil, 2015.

${ }^{24}$ Voir notamment Philip Nord, «Pierre Schaeffer and Jeune France: Cultural Politics in the Vichy Years », French Historical Studies, Vol. 30, No. 4 (Fall 2007), p. 865-709.

25 Olivier Gariguel, Panorama des revues littéraires sous l'Occupation, Paris, Imec, 2007, p. 369.

${ }^{26}$ Sous le nom de Chris Mayor, Esprit, mai 1946, p. 768-785.

${ }^{27}$ Paul Flamand, Fiche de lecture D'une planète morte, mai/juin 1946.

28 On relève plus de 70 contributions de toutes tailles et de toutes natures entre 1946 et 1955. Pour la bibliographie de Marker, on renvoie à l'excellent travail de recensement et de commentaires fait par les animateurs du site http://www.chrismarker.ch/index.html [consulté le 17 juillet 2015].

${ }^{29}$ Chris Mayor, Esprit, octobre 1946, p. 511-513.

${ }^{30}$ Catherine Lupton, Chris Marker. Memories of the Future, Londres, Reaktion Books, 2005, chapitre 1.

${ }^{31}$ Guy Dumur, « Souvenirs de Giraudoux », Notre temps, 26 juillet 1952.

${ }^{32}$ Christian Marker, Giraudoux par lui-même, Paris, Seuil, 1952, p. 50.

${ }^{33}$ Lettre de Paul Flamand à Jean de La Varende, 27 avril 1950.

34 A.G., Monde Nouveau, nº 66, 1952; et Beaux-Arts, 26 septembre 1952.

35 Gabriel Venaissin, "Jean Giraudoux avec nous ou derrière nous », La Vie intellectuelle, mars 1952, p. 118.

${ }^{36}$ Christian Marker, Giraudoux par lui-même, Paris, Seuil, 1952, p. 10.

37 André Bazin, Esprit, juillet 1952, p. 162-163.

Vol. 7, $n^{\circ} 1 \mid$ Automne 2015

«Une fabrique collective du patrimoine littéraire (XIX ${ }^{\mathrm{e}}-\mathrm{XXI}^{\mathrm{e}}$ siècles). Les collections de 
${ }^{38}$ Jean Mauduit, Témoignage chrétien, 11 juillet 1952.

${ }^{39}$ Dès l'introduction de son essai, Marker relève que «par quelque bout que l'on prenne Giraudoux, il est le premier»: de sa classe, au Concours général et à Normale, aux concours des Chancelleries et durant les compétitions sportives auxquelles il s'adonne, premier écrivain décoré pendant la guerre de 1914-1918, premier écrivain engagé, intéressé par le cinéma en écrivain, ou encore remarqué par Gide pour son premier livre... Un rang qui, probablement, le distingue de la décadence de son époque?

${ }^{40}$ Christian Marker, Giraudoux par lui-même, Paris, Seuil, 1952, p. 34.

${ }^{41}$ André Bazin, Esprit, juillet 1952, p. 162-163.

${ }^{42}$ Christian Marker, Giraudoux par lui-même, Paris, Seuil, 1952, p. 34.

${ }^{43}$ René Pomeau, avant-propos du numéro "Giraudoux en son temps », Revue d'histoire littéraire de la France, 5/6, septembre-décembre 1983, p. 707 (p. 707-710). Sur Giraudoux : Philippe Dufay, Jean Giraudoux: biographie, Paris, Julliard, 1983; et Jacques Body, Jean Giraudoux, Paris, Gallimard, 2004.

${ }^{44}$ François-Georges Dreyfus, «Un non-conformiste des années trente : Giraudoux et la politique », Revue d'histoire littéraire de la France, 5/6, septembre-décembre 1983, p. 725-734.

45 Pierre Vidal Naquet, «Sur Pleins pouvoirs», Cahiers Jean Giraudoux, n² 21, 1992, p. 17-26. Dans le même volume, Jeanine Verdès-Leroux, "Un faux-pas? À propos de Pleins pouvoirs», p. 27-39. Voir aussi Pierre D’Almeida, "La responsabilité de l'écrivain : Giraudoux, d'une guerre l'autre », Cabiers Jean Giraudoux, n 36, textes réunis par Sylviane Coyault, 2008, p. 352-362.

46 Des travaux permettent de situer cette nomination. Cf. Didier Georgakakis, «La République contre la propagande d'État? Création et échec du Commissariat général à l'information (juillet 1939-avril 1940)", Revue française de science politique, vol. 48, $\mathrm{n}^{\circ}$ 5, octobre 1998, p. 606-624. Également, Guillaume Marceau, «Jean Giraudoux, un écrivaindiplomate à la tête de la Propagande d'État (1939-1940) », Julien Massicotte, Maria Neagu, Stéphane Savard (dir.), Actes du 7e colloque du Département d'bistoire de l'Université Laval, 2007, Québec, Artefact, p. 95-110.

${ }^{47}$ François Chaslin, Un Corbusier, Paris, Seuil, 2015, p. 173 sq.

${ }^{48}$ Christian Marker, Giraudoux par lui-même, Paris, Seuil, 1952, p. 30.

${ }^{49}$ Christian Marker, Giraudoux par lui-même, Paris, Seuil, 1952, p. 34.

${ }^{50}$ Christian Marker, Giraudoux par lui-même, Paris, Seuil, 1952, p. 36.

${ }^{51}$ Christian Marker, Giraudoux par lui-même, Paris, Seuil, 1952, p. 41-42.

52 Il faudrait encore reprendre la lecture de Marker pour saisir la manière dont il s'appuie sur Louis Aragon (celui des Communistes) afin de légitimer l'idée d'un Giraudoux « engagé ».

Vol. 7, $\mathrm{n}^{\circ} 1 \mid$ Automne 2015

«Une fabrique collective du patrimoine littéraire (XIX ${ }^{\mathrm{e}}-\mathrm{XXI}^{\mathrm{e}}$ siècles). Les collections de 
${ }^{53}$ Selon l'expression de Christian Allegre, "Giraudoux », Jean-Claude Polet (dir.), Auteurs européens du premier XXe siècle : 2. Cérémonial pour la mort du sphynx (1940-1958), Bruxelles, De Boeck, 2002, p. 285-287.

${ }^{54}$ Anna Boschetti, Sartre et «Les Temps Modernes », Paris, Minuit, 1985.

${ }^{55}$ Guy Dumur, « Giraudoux, les anges et les hommes », Combat, 19 juin 1952.

56 Annie Cohen-Solal, Sartre. 1905-1980, Paris, Gallimard, 1985, p. 85-86.

${ }^{57}$ Anna Boschetti, Sartre et «Les Temps Modernes », Paris, Minuit, 1985, p. 31.

58 Anna Boschetti, Sartre et «Les Temps Modernes », Paris, Minuit, 1985, p. 63.

59 Article repris dans Jean-Paul Sartre, Situations I. Essais critiques, Paris, Gallimard, 1947, p. 76-91. Je m'appuie ici sur plusieurs notices de François Noudelmann, Gilles Philippe (dir.), Dictionnaire Sartre, Paris, Honoré Champion, 2004; en particulier Paolo Tamassia, «M. Jean Giraudoux et la philosophie d'Aristote. À propos de Choix des élues», p. 324-325, et Jacques Deguy, «Jean Giraudoux », p. 200.

60 Alain Virmaux, "Cinéma », et Sandra Teroni, "Citizen Kane », in François Noudelmann, Gilles Philippe (dir.), Dictionnaire Sartre, Paris, Honoré Champion, 2004, p. 89-91. En 1950, André Bazin consacre son premier livre à Citizen Kane.

${ }^{61}$ Christian Marker, Giraudoux, Paris, Seuil, 1952, p. 17-22.

62 Philippe Olivera, "Aragon, réaliste socialiste. Les usages d'une étiquette littéraire des années Trente aux années Soixante », Sociétés et Représentations, 2003/1, nº 15, p. 229-246.

${ }^{63}$ Chris Marker, «Les derniers feux du roman», Esprit, avril 1947, p. 703-707. Cette recension de plusieurs romans, dont les grandes lignes sont reprises dans le Giraudoux, est l'occasion pour Marker de développer cette fin du roman, perdu dans l'individualisme de la représentation, au profit, peut-être, d'un "nouveau lyrisme » inspiré par Lautréamont, les surréalistes, les primitifs et Antonin Artaud, « peut-être le premier classique ».

${ }^{64}$ Christian Marker, Giraudoux, Paris, Seuil, 1952., p. 27-29.

${ }^{65}$ Christian Marker, Giraudoux, Paris, Seuil, 1952, p. 30-31.

${ }^{66}$ Christian Marker, Giraudoux, Paris, Seuil, 1952, p. 43.

${ }^{67}$ Outre ce dossier de presse, les informations disponibles révèlent que, sur le long terme, ce livre de Marker connaît une vente dans la moyenne haute de la collection.

68 Pascal Fouché, «L'Édition. 1914-1992», Histoire des droites en France. t. 2. Cultures, Paris, Gallimard, 1992, p. 257-292.

${ }^{69}$ Bien entendu, les rééditions du livre ne suscitent quasiment pas de réception critique.

Vol. $7, n^{\circ} 1 \mid$ Automne 2015

«Une fabrique collective du patrimoine littéraire (XIX ${ }^{\mathrm{e}}-\mathrm{XXI}^{\mathrm{e}}$ siècles). Les collections de 
${ }^{70}$ Chris Marker s'appuie à plusieurs reprises sur le livre de Claude-Edmonde Magny, jusqu'à affirmer qu'il « faudrait [le] citer tout le temps »; Giraudoux par lui-même, Paris, Seuil, 1952, p. 42.

${ }^{71}$ Cf. Pierre-Aimé Touchard, Esprit, mars 1945, p. 602-604.

${ }^{72}$ Claude-Edmonde Magny, «Permanence de Giraudoux », Samedi Soir, 26 juillet 1957.

73 Albert Béguin, "Où en sommes-nous avec Jean Giraudoux », Gazette de Lausanne, 14 juin 1952.

${ }^{74}$ André Bazin, Esprit, juillet 1952, p. 162-163.

${ }^{75}$ Claude Roy, « Aimer Giraudoux », Libération, 23 juillet 1952.

${ }^{76}$ André Séailles, «Le retour de Jean Giraudoux », Table Ronde, septembre 1952, p. 151 156.

${ }^{77}$ Jean Mauduit, « Giraudoux par lui-même », Témoignage Chrétien, 11 juillet 1952.

78 Gabriel Venaissin, «Jean Giraudoux avec nous ou derrière nous », La Vie intellectuelle, mars 1952, p. 117-121.

79 Pierre de Boisdeffre, Études, novembre 1952.

80 Anna Boschetti, Sartre et «Les Temps modernes », Paris, Minuit, 1985, p. 35.

${ }^{81}$ Un travail systématique de comparaison des textes et des illustrations apporterait des indications. Il doit, comme nous avons commencé à le faire, s'accompagner d'une mise en perspective du Girandoux de Marker avec ses articles dans Esprit.

82 Je dois ce constat et les informations précieuses qui l'établissent à Mathilde Labbé, que je remercie vivement pour me les avoir communiquées.

83 Conclusion d'un entretien avec Jean-Louis Pays [Jean-Louis Chichin], Miroir du cinéma, n² 2, mai 1962 (cf. http://www.chrismarker.ch/index.html).

${ }^{84}$ En témoignent certains motifs du refus de publication opposé à Samuel Beckett en 1947 : voir Hervé Serry, «A Publishing Decision under Constraint. Samuel Beckett and le Seuil Publishers in 1947 », Journal of Beckett Studies, vol. 21, issue 1 (traduit par Helena Scott), 2012, p. 65-87; repris dans Littérature (Paris, Larousse), n 167, octobre 2012, p. 51 64.

${ }^{85}$ Sur cette réappropriation par Sartre, depuis une philosophie de la liberté, voir Gisèle Sapiro, La Guerre des écrivains, Paris, Fayard, p. 599 sq.

Vol. 7, $\mathrm{n}^{\circ} 1 \mid$ Automne 2015

«Une fabrique collective du patrimoine littéraire (XIX ${ }^{\mathrm{e}}-\mathrm{XXI}^{\mathrm{e}}$ siècles). Les collections de monographies illustrées » 


\section{Bibliographie}

Christian Allegre, "Giraudoux », Jean-Claude Polet (dir.), Auteurs européens du premier XXe siècle : 2. Cérémonial pour la mort du sphynx (1940-1958), Bruxelles, De Boeck, 2002, p. 285-287.

Hasan Anamur, Bibliographie chronologique des publications de langue française sur Jean Giraudoux et son oeuvre de 1909 à 1970, Ankara, Université d'Ankara, 1980.

Jacques Body, Jean Giraudoux, Paris, Gallimard, 2004.

Anna Boschetti, Sartre et «Les Temps Modernes », Paris, Minuit, 1985.

Olivier Cariguel, Panorama des revues littéraires sous l'Occupation, 1939-1945, Paris, Imec, 2007.

Annie Cohen-Solal, Sartre. 1905-1980, Paris, Gallimard, 1985.

François Chaslin, Un Corbusier, Paris, Seuil, 2015.

Pierre D'Almeida, «La responsabilité de l'écrivain : Giraudoux, d'une guerre l'autre ", Cabiers Jean Giraudoux, n 36, textes réunis par Sylviane Coyault, 2008, p. $352-362$.

Jacques Deguy, «Jean Giraudoux », François Noudelmann et Gilles Philippe (dir.), Dictionnaire Sartre, Paris, Honoré Champion, 2004, p. 200.

François-Georges Dreyfus, «Un non-conformiste des années trente : Giraudoux et la politique ", Revue d'bistoire littéraire de la France, 5/6, septembre-décembre 1983, p. $725-734$.

Philippe Dufay, Jean Giraudoux : biographie, Paris, Julliard, 1983.

Pascal Fouché, «L'Édition. 1914-1992 », Histoire des droites en France. t. 2. Cultures, Paris, Gallimard, 1992, p. 257-292.

Didier Georgakakis, «La République contre la propagande d'État? Création et échec du Commissariat général à l'information (juillet 1939-avril 1940) », Revue française de science politique, vol. 48, n 5, octobre 1998, p. 606-624.

François Lecointe, «Tombeau pour Chris Marker», Esprit, 18 octobre 2012. http:/ / www.esprit.presse.fr/news/ frontpage/ news.php?code $=232$ 
Catherine Lupton, Chris Marker. Memories of the Future, Londres, Reaktion Books, 2005.

Guillaume Marceau, «Jean Giraudoux, un écrivain-diplomate à la tête de la Propagande d'État (1939-1940) », Julien Massicotte, Maria Neagu, Stéphane Savard (dir.), Actes du 7e colloque du Département d'histoire de l'Université Laval, 2007, Québec, Artefact, p. 95-110.

David Martens, «L'exercice pseudonymique de l'autorité littéraire. Un partage des voix contesté », Emmanuel Bouju (dir.), L'autorité en littérature: exercice, partage, contestation, Rennes, PUR, 2010, p. 237-247.

Chris Marker, Giraudoux par lui-même, Paris, Seuil, 1952.

Chris Marker, Jean Giraudoux in Selbstzengnissen und Bilddokumenten, Hambourg, Rowohlt, 1962.

Chris Marker, Marker Mémoire, Paris, Cinémathèque française, janvier-février 1998.

Philip Nord, «Pierre Schaeffer and Jeune France: Cultural Politics in the Vichy Years », French Historical Studies, Vol. 30, No. 4 (Fall 2007), p. 865-709.

Philippe Olivera, «Aragon, réaliste socialiste. Les usages d'une étiquette littéraire des années Trente aux années Soixante », Sociétés et Représentations, 2003/1, n 15, p. 229-246.

Bernard Pingaud, Inventaire, Paris, Gallimard, 1965.

Bernard Pingaud, Une tâche sans fin. Mémoires, Seuil, 2009.

Bamchade Pourvali, «Jeunesses de Chris Marker», Vertigo, 2013/2, n 46, p. 7-14.

Gisèle Sapiro, La Guerre des écrivains, Paris, Fayard, 1999.

Jean-Paul Sartre, Situations I. Essais critiques, Paris, Gallimard, 1947.

Hervé Serry, Les Éditions du Senil. 70 ans d'histoire, Paris, Seuil, 2008.

Hervé Serry, "A Publishing Decision under Constraint. Samuel Beckett and le Seuil Publishers in 1947 », Journal of Beckett Studies, vol. 21, issue 1 (traduit par Helena Scott), 2012, p. 65-87. Repris en français dans Littérature (Paris, Larousse), $n^{\circ} 167$, octobre 2012, p. 51-64.

Hervé Serry, Aux Origines du Seuil, Paris, Seuil, 2015.

Vol. 7, $\mathrm{n}^{\circ} 1 \mid$ Automne 2015

«Une fabrique collective du patrimoine littéraire (XIX ${ }^{\mathrm{e}}-\mathrm{XXI}^{\mathrm{e}}$ siècles). Les collections de 
Hervé Serry, «Une "vulgarisation de qualité" aux Éditions du Seuil. Conditions éditoriales pour la diffusion de nouvelles formes de savoirs (1945-1965)», L. Braida et B. Ouvry-Vial (dir.), Textes, Formes, Lectures en Europe $\left(18^{\mathrm{e}}-21^{\mathrm{e}}\right), 2016$ (à paraître).

Simone Signoret, La Nostalgie n'est plus ce qu'elle était, Paris, Seuil, 1976.

Paolo Tamassia, «M. Jean Giraudoux et la philosophie d'Aristote. À propos de Choix des élues», François Noudelmann, Gilles Philippe (dir.), Dictionnaire Sartre, Paris, Honoré Champion, 2004, p. 324-325.

Jeanine Verdès-Leroux, "Un faux-pas? À propos de Pleins pouvoirs», Cabiers Jean Giraudoux, n 21, 1992, p. 27-39.

Pierre Vidal Naquet, "Sur Pleins pouvoirs », Cabiers Jean Girandoux, n 21, 1992, p. $17-26$.

Alain Virmaux, "Cinéma », et Sandra Teroni, "Citizen Kane », François Noudelmann, Gilles Philippe (dir.), Dictionnaire Sartre, Paris, Honoré Champion, 2004, p. 89-91.

Vol. 7, $\mathrm{n}^{\circ} 1 \mid$ Automne 2015

«Une fabrique collective du patrimoine littéraire (XIX ${ }^{\mathrm{e}}-\mathrm{XXI}^{\mathrm{e}}$ siècles). Les collections de monographies illustrées » 